\title{
Methylprednisolone pulse therapy combined with mizoribine following tonsillectomy for immunoglobulin A nephropathy: clinical remission rate, steroid sparing effect, and maintenance of renal function
}

\author{
Tomohiro Kaneko $\cdot$ Akio Hirama $\cdot$ Kae Ueda \\ Teppei Fujino $\cdot$ Koichi Utsumi $\cdot$ Yasuhiko Iino • \\ Yasuo Katayama
}

Received: 26 June 2010/ Accepted: 19 September 2010/Published online: 23 October 2010

(C) Japanese Society of Nephrology 2010

\begin{abstract}
Background The long-term prognosis of immunoglobulin A nephropathy (IgAN) is reportedly poor. In Japan, tonsillectomy-steroid pulse therapy has frequently been used for treatment of early IgAN, with favorable outcomes. However, steroid usage is sometimes limited due to adverse reactions. To reduce the total dose of steroids, we have been using mizoribine (MZR) in combination with tonsillectomy-steroid pulse therapy since 2004. Here we report a retrospective evaluation of our protocol outcome. Methods Forty-two patients aged $<70$ years with histopathologically confirmed IgAN and an estimated glomerular filtration rate (eGFR) of $30 \mathrm{ml} / \mathrm{min} / 1.73 \mathrm{~m}^{2}$ or higher were enrolled. After giving informed consent, all the patients underwent bilateral tonsillectomy. One week later, intravenous methylprednisolone pulse therapy $(500 \mathrm{mg} /$ day $)$ was administered for 3 days, followed by oral prednisolone ( $30 \mathrm{mg} /$ day and tapered to 0 over 7 months) and MZR (150 mg/day for 11 months). The complete remission (CR) rate and renoprotective effect were assessed.

Results The CR rate at 6, 12, and 24 months was 33.3, 69.1 , and $76.2 \%$, respectively. Despite a relatively low total steroid dose, renal function was satisfactorily maintained for 24 months or longer with no relapse. The eGFR in patients with stage 3 chronic kidney disease was significantly improved at 6 months after start of treatment. Three patients $(7.1 \%)$ had mild and transient adverse events.
\end{abstract}

T. Kaneko $(\bowtie) \cdot$ A. Hirama $\cdot$ K. Ueda $\cdot$ T. Fujino .

K. Utsumi · Y. Iino · Y. Katayama

Divisions of Neurology, Nephrology and Rheumatology,

Department of Internal Medicine, Nippon Medical School,

1-1-5 Sendagi, Bunkyo-ku, Tokyo 113-8603, Japan

e-mail: tomohiro@nms.ac.jp
Conclusion This protocol appears to be highly effective and safe for IgAN patients with renal dysfunction.

Keywords Clinical remission · IgA nephropathy · Methylprednisolone pulse $\cdot$ Mizoribine ·

Steroid sparing effect · Tonsillectomy

\section{Introduction}

Immunoglobulin A nephropathy (IgAN) was first described by Berger et al. [1]. Approximately $40 \%$ of IgAN patients develop renal failure within 20 years of diagnosis, and the long-term prognosis is poor [2]. Pozzi et al. [3] reported that corticosteroid therapy for IgAN exerted a renoprotective effect, but that relapse of proteinuria was observed in a relatively large number of patients after treatment. This report also suggested that complete remission (CR) cannot be achieved without preventing continuous tissue deposition of IgA.

Focal infection of the palatine tonsils or other mucosal sites causes immune abnormalities, leading to sugar-chain incompleteness in $\operatorname{IgA} 1$, which is then overproduced and deposited in renal glomeruli [4]. In Japan, high rates of CR have been reported in patients with early IgAN after bilateral palatine tonsillectomy and steroid pulse therapy $[5,6]$. In some patients, however, steroid-associated adverse events have occurred in a dose-dependent manner, necessitating dose reduction. An increase in the number of sclerotic glomeruli as well as in the degree of interstitial fibrosis due to steroid therapy has also been reported in patients with low glomerular filtration rates (GFRs) [7].

Mizoribine (MZR) is an immunosuppressive agent used for the treatment of nephrotic syndrome caused by primary glomerulonephritis. A decrease in the intensity of $\operatorname{IgA}$ 
staining in glomerular mesangial areas, as well as a decrease in the number of B cells and IgA-bearing B cells, has been demonstrated in a MZR-treated animal model of IgAN [8]. In another study involving 34 children with diffuse IgAN who received steroid pulse therapy in combination with MZR, there was a significant decrease in the degree of $\operatorname{IgA}$ deposition and infiltration of the glomeruli by CD68-positive cells and alpha-smooth muscle actinpositive cells, and consequently a decrease in the extent of tissue damage [9]. Other reports have also indicated that MZR ameliorates glomerular sclerosis and tubulointerstitial fibrosis [10, 11].

To reduce the total dose of steroids, since 2004 we have been using MZR for IgAN in combination with tonsillectomy and steroid pulse therapy. Specifically, patients receive one course of steroid pulse therapy instead of the current three courses and postoperative oral steroid therapy for 7 months instead of 11 months, in combination with MZR. In the present study, data from 42 patients followed up for at least 24 months were used to determine the rate of CR (assessed by urinalysis), the treatment efficacy in protecting against renal function deterioration, and the safety of the therapy.

\section{Patients and methods}

\section{Patients}

We retrospectively assessed the therapeutic outcome of MZR in combination with tonsillectomy plus steroid pulse therapy in 42 patients who fulfilled the following eligibility criteria and were followed up for at least 2 years: (1) IgAN diagnosed by renal biopsy, with grade 2 , 3 , or 4 histologic lesions [12]; (2) persistent microscopic hematuria and proteinuria; (3) a serum creatinine level of $<2.0 \mathrm{mg} / \mathrm{dl}$; (4) an estimated glomerular filtration rate (eGFR) of $30 \mathrm{ml} / \mathrm{min} / 1.73 \mathrm{~m}^{2}$ or higher; (5) age $<70$ years; and (6) willingness to provide written informed consent.

We excluded patients for whom steroids were contraindicated and also those in whom the renal disease was associated with systemic lupus erythematosus or other systemic diseases. Patients whose urinary protein/ creatinine ratio was less than $0.1(\mathrm{~g} / \mathrm{g})$ were also excluded from the study.

\section{Therapeutic intervention}

After obtaining informed consent, bilateral palatine tonsillectomy was performed on all patients. One week after surgery, intravenous methylprednisolone (mPSL) pulse therapy (500 mg/day) was administered for 3 days, and each patient was started on an antiplatelet drug (dilazep hydrochloride or dipyridamole), an anti-ulcer drug, and sulfamethoxazole-trimethoprim (SMX-TMP). After the mPSL pulse therapy, the patients continued to receive oral prednisolone (PSL) at a dose of $30 \mathrm{mg}$ per day for 4 weeks, and then once every 2 days thereafter in combination with MZR at $150 \mathrm{mg} /$ day once daily. The dose of PSL was then decreased by $5 \mathrm{mg}$ every 4 weeks and discontinued in the 7 th month. SMX-TMP and the anti-ulcer drug were discontinued, in principle, when the PSL dose was $20 \mathrm{mg}$ administered every 2 days. MZR and the antiplatelet drug were continued for 11 months (Fig. 1). Patients with hypertension received an antihypertensive drug such as a renin-angiotensin system inhibitor.

\section{Efficacy assessment}

The primary endpoint was the rate of CR (assessed by urinalysis at 6,12 , and 24 months for proteinuria and hematuria). CR was defined as remission of both proteinuria and hematuria, specifically, (1) a protein/creatinine ratio of $<0.1(\mathrm{~g} / \mathrm{g})$ for at least 3 months, after correction of urinary protein levels for urinary creatinine concentrations, and (2) $<5$ red blood cells per high-power field on microscopic evaluation of the urinary sediment.

The secondary endpoint was the efficacy of our treatment in preventing progressive deterioration of $\operatorname{IgAN}$, which was assessed by evaluation of renal function and immunological investigations. The eGFR was calculated using the equation recommended by the Japanese Society of Nephrology: $\mathrm{eGFR}=194 \times \mathrm{sCr}^{-1.094} \times \operatorname{age}^{-0.287}(\times 0.739$ if female $)$ [13], and patients were categorized into three stages of chronic kidney disease (CKD) based on the eGFR values. The immunological parameters evaluated were the serum levels of $\operatorname{IgA}$ and IgG. When possible, urinary IgE levels were also measured along with the urinary levels of interleukin-6 (IL-6) using a highly sensitive IL-6 assay.

The presence or absence of adverse events was examined during the follow-up period by periodic determination of blood pressure, hematological and biochemical parameters, urinalysis, and infection markers.

\section{Statistics}

Statistical analysis was conducted by examination of normality, and the results were compared using the Wilcoxon rank sum test. The uniformity of process variables was analyzed by the chi-squared $\left(\chi^{2}\right)$ test. To test the equality of the means, repeated-measures ANOVA was employed. The statistical significance level was set at $P<0.05$ (for a twotailed test). The statistical software package SPSS for Microsoft Windows version 11.0 (SPSS Inc., Chicago, IL) was used for the analyses. The mean values and standard deviations were calculated for data representation. 


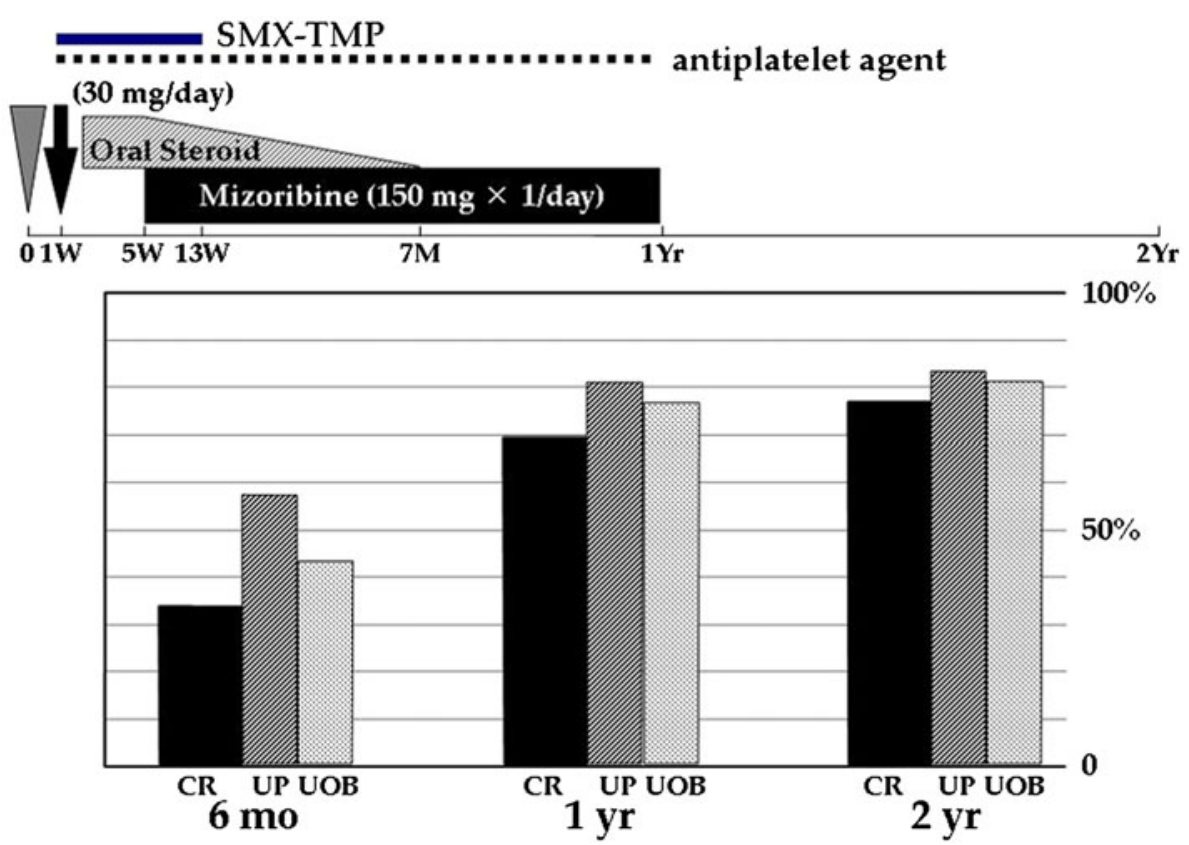

Fig. 1 Tonsillectomy plus steroid pulse + oral steroid + mizoribine therapy protocol: time-course change in rates of CR of IgAN (rates of remission of proteinuria and hematuria). Therapy was started (0) at the time of tonsillectomy (inverted triangle); 1 week later, one course of methylprednisolone pulse therapy (downward arrow) was administered. Sulfamethoxazole-trimethoprim (SMX-TMP), an antiplatelet drug, and an anti-ulcer drug were administered. An oral steroid was then administered at a dose of $30 \mathrm{mg}$ daily for 4 weeks, and then once every 2 days in combination with MZR at a dose of $150 \mathrm{mg}$ once

\section{Results}

Table 1 shows the baseline characteristics of the patients according to CKD stage. The duration of illness (from detection of urinalysis abnormalities to tonsillectomy) averaged $5.7 \pm 4.8$ (0.4-20) years (unknown in 3 cases). The duration of illness tended to be longer in elderly patients. The proportion of RAS inhibitor users was $38 \%$. This percentage rose significantly as CKD stage became higher. No significant change in blood pressure was observed during the treatment, and none of the patients required the use of additional antihypertensive medication after the start of the therapy.

The rate of CR for the 42 patients after tonsillectomy and steroid pulse therapy plus MZR therapy was $33.3 \%$ $(n=14)$ at 6 months, $69.1 \%(n=29)$ at 1 year, and $76.2 \%(n=32)$ at 2 years. In many patients, improvement of proteinuria preceded the improvement of hematuria (Fig. 1). No patients showed relapse of IgAN during the follow-up period (average of $2.65 \pm 1.03$ years) after obtaining CR.

Overall, there were no significant changes in the eGFR during the follow-up period. Analysis by CKD stage daily. MZR was administered for 11 months, and the antiplatelet drug was administered for 12 months. SMX-TMP and the anti-ulcer drug were administered for 13 weeks and then discontinued. The dose of the steroid was gradually reduced until the end of 7 months, and then discontinued. The rates of CR of IgAN were determined as the rates of remission of proteinuria and hematuria at 6,12 , and 24 months. The number of patients was 42 . $C R$ complete remission, $U P$ urinary protein, $U O B$ urine occult blood

showed that eGFR remained unchanged in patients with CKD stages 1 and 2, but was significantly improved in patients with CKD stage 3 at 6 months and later after the start of treatment (Fig. 2).

Table 2 shows the changes in urinary protein excretion and laboratory values. Compared with the baseline value, a significant decrease in urinary protein excretion was observed at 6, 12, and 24 months, and a significant decrease in serum creatinine levels was evident at 12 and 24 months.

Compared with the baseline value, a significant decrease in serum IgA levels was observed at 6, 12, and 24 months. While a significant decrease in the serum levels of $\mathrm{IgG}$ and $\mathrm{IgE}$ was observed at 6 and 12 months, these parameters returned almost to baseline levels at 24 months. Compared with the baseline value, there was a significant decrease in the urinary levels of IL-6 at 6, 12, and 24 months.

During the follow-up period, steroid-induced acne occurred in three patients as an adverse event and required treatment, although this was transient. Except for pain, there were no other tonsillectomy-related complications. None of the patients developed severe immunosuppression 
Table 1 Baseline characteristics of patients according to CKD stage

\begin{tabular}{|c|c|c|c|c|}
\hline Characteristic & All $(n=42)$ & CKD stage $1(n=18)$ & CKD stage $2(n=16)$ & CKD stage $3(n=8)$ \\
\hline Age (years) & $30.4 \pm 11.9$ & $22.6 \pm 4.9$ & $31.9 \pm 6.2$ & $45.0 \pm 16.8$ \\
\hline Gender $(\mathrm{M} / \mathrm{F})$ & $17 / 25$ & $6 / 12$ & $8 / 8$ & $3 / 6$ \\
\hline Urine $\mathrm{OB}$ score & $2.60 \pm 0.59$ & $2.78 \pm 0.43$ & $2.31 \pm 0.70$ & $2.75 \pm 0.46$ \\
\hline Proteinuria $(\mathrm{g} / \mathrm{g} \times \mathrm{Cre})$ & $0.98 \pm 0.98$ & $0.73 \pm 0.68$ & $0.72 \pm 0.59$ & $2.03 \pm 1.49$ \\
\hline No. of patients with UP $>1.0 \mathrm{~g} / \mathrm{g} \times$ Cre & $17(40.5 \%)$ & $7(38.9 \%)$ & $5(31.3 \%)$ & $5(62.5 \%)$ \\
\hline $\mathrm{eGFR}\left(\mathrm{ml} / \mathrm{min} / 1.73 \mathrm{~m}^{2}\right)$ & $85.0 \pm 27.7$ & $111.6 \pm 12.5$ & $75.3 \pm 8.6$ & $44.8 \pm 8.4$ \\
\hline Serum creatinine $(\mathrm{mg} / \mathrm{dl})$ & $0.83 \pm 0.27$ & $0.62 \pm 0.09$ & $0.86 \pm 0.16$ & $1.24 \pm 0.22$ \\
\hline Serum IgA (mg/dl) & $360.1 \pm 134.4$ & $309.1 \pm 93.3$ & $371.1 \pm 133.5$ & $447.9 \pm 172.4$ \\
\hline Serum IgE (IU/ml) & $439.2 \pm 670.9$ & $338.1 \pm 331.3$ & $608.7 \pm 1000.2$ & $322.8 \pm 413.1$ \\
\hline Serum IgG (mg/dl) & $1207.9 \pm 292.4$ & $1330.7 \pm 303.8$ & $1136.5 \pm 224.9$ & $1093.6 \pm 315.5$ \\
\hline Urine HS IL-6 (pg/ml) & $10.58 \pm 17.26$ & $8.76 \pm 9.31$ & $13.09 \pm 25.47$ & $9.50 \pm 9.60$ \\
\hline Duration of illness (years) & $5.7 \pm 4.8$ & $5.6 \pm 4.3$ & $5.4 \pm 5.1$ & $7.2 \pm 5.8$ \\
\hline \multicolumn{5}{|l|}{ Histological grade } \\
\hline 1 & $0(0 \%)$ & 0 & 0 & 0 \\
\hline 2 & $22(52.4 \%)$ & 13 & 8 & 1 \\
\hline 3 & $17(40.5 \%)$ & 5 & 8 & 4 \\
\hline 4 & $3(7.1 \%)$ & 0 & 0 & 3 \\
\hline No. of RAS inhibitor users & 16 & 3 & 5 & 8 \\
\hline $\mathrm{SBP}(\mathrm{mmHg})$ & $116.05 \pm 12.07$ & $112.11 \pm 8.90$ & $115.88 \pm 11.79$ & $125.25 \pm 15.04$ \\
\hline DBP $(\mathrm{mmHg})$ & $68.10 \pm 10.42$ & $66.00 \pm 10.78$ & $67.63 \pm 8.86$ & $73.35 \pm 11.68$ \\
\hline
\end{tabular}

No. of patients (percentage of patients). For continuous variables, mean \pm standard deviation

$O B$ occult blood, $U P$ urinary protein, $e G F R$ estimated glomerular filtration rate, HS IL-6 highly sensitive interleukin 6, SBP systolic blood pressure, $D B P$ diastolic blood pressure

Fig. 2 Time-course changes in glomerular filtration rate (GFR). GFR in patients by CKD stages 1 (filled circles), 2 (filled triangles), and 3 (filled squares); mean values $\pm \mathrm{SD}$. $* P<0.05$ (compared with baseline): Wilcoxon's rank sum test. The number of patients in parentheses

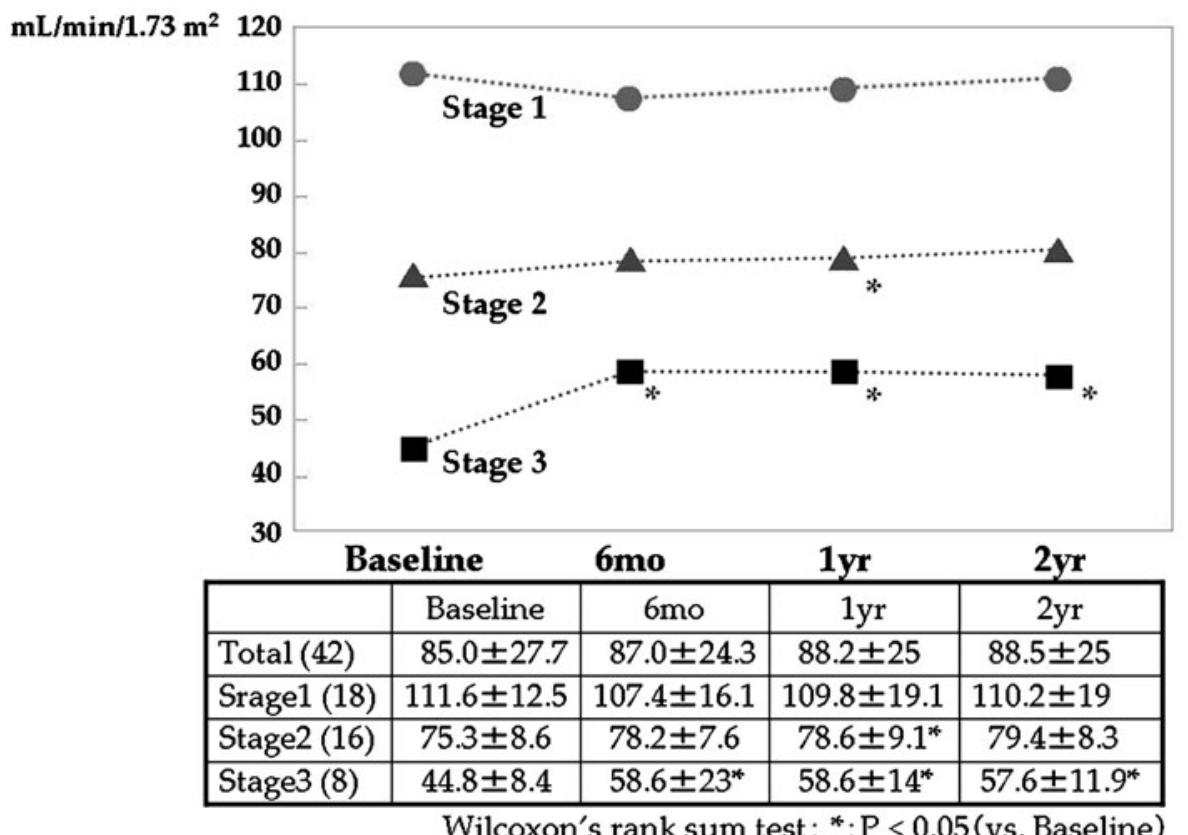

Wilcoxon's rank sum test: *:P $<0.05$ (vs. Baseline)
(CD4 $<400 \%$, IgG $<600 \mathrm{mg} / \mathrm{dl}$ ) or other severe adverse events such as infections, diabetes, aggravated hypertension, psychiatric symptoms, hyperuricemia, or serious changes in laboratory values (data not shown).

\section{Discussion}

In this observational study, we investigated the long-term efficacy and safety and steroid-sparing effect of 
Table 2 Time course changes in urinary protein excretion and laboratory values

\begin{tabular}{lcccc}
\hline & Baseline & 6 months & 1 year & 2 years \\
\hline Urinary protein $(\mathrm{g} / \mathrm{g} \mathrm{Cr})$ & $0.98 \pm 0.98$ & $0.24 \pm 0.62^{* * *}$ & $0.12 \pm 0.51^{* * *}$ & $0.09 \pm 0.22^{* * *}$ \\
Serum creatinine $(\mathrm{mg} / \mathrm{dl})$ & $0.83 \pm 0.27$ & $0.80 \pm 0.22$ & $0.77 \pm 0.19^{* *}$ & $0.76 \pm 0.19^{* *}$ \\
IgA $(\mathrm{mg} / \mathrm{dl})$ & $360.1 \pm 134.4$ & $283.3 \pm 90.9 * * *$ & $230.0 \pm 97.2^{* * *}$ & $257.2 \pm 122.2^{* * *}$ \\
IgG $(\mathrm{mg} / \mathrm{dl})$ & $1207.9 \pm 292.4$ & $799.0 \pm 200.7 * * *$ & $1008.3 \pm 253.2^{* * *}$ & $1064.1 \pm 205.9$ \\
IgE $(\mathrm{IU} / \mathrm{ml})$ & $439.2 \pm 670.9$ & $299.9 \pm 372.2^{*}$ & $122.3 \pm 130.3 * * *$ & $374.4 \pm 450.6$ \\
HS IL-6 (pg/ml) & $10.6 \pm 17.3$ & $6.1 \pm 7.4 * *$ & $3.0 \pm 5.1^{* * *}$ & $4.4 \pm 7.1 * *$ \\
\hline
\end{tabular}

Wilcoxon's rank sum test; $* P<0.05, * * P<0.01, * * * P<0.001$

HS IL-6 highly sensitive interleukin 6

tonsillectomy-steroid pulse therapy in combination with MZR in IgAN patients with stage 1-3 CKD. The rate of $\mathrm{CR}$, assessed by urinalysis, was $69.1 \%$ at 12 months, increasing to $76.2 \%$ at 24 months.

In recent years, IgAN patients who undergo palatine tonsillectomy to treat focal infection of the palatine tonsils have been given steroid pulse therapy to prevent recurrence of IgAN (three courses of mPSL therapy and 1 year of oral steroid therapy). Reported rates of CR for this treatment vary depending on the definition used. Hotta et al. [5] reported that urinary abnormalities disappeared in $48 \%$ of the patients receiving this treatment and that no patients showed progressive deterioration. Komatsu et al. [6] compared tonsillectomy plus steroid pulse therapy (one course of steroid pulse therapy and 18 months of oral steroid therapy) with steroid pulse monotherapy, and found that the former treatment was significantly more effective than the latter, with a CR rate of $61.8 \%$ at 24 months. At the baseline, $37.1 \%$ of patients in that study had a urinary protein excretion of more than $1000 \mathrm{mg}$, and $8.6 \%$ had a serum creatinine level exceeding $1.2 \mathrm{mg} / \mathrm{dl}$. The patient baseline characteristics and the histological severity of the disease in that study were markedly similar to those in our study. Their findings reliably reflect the prognosis of IgAN and are useful for comparative assessment of clinical efficacy.

Since a control group without MZR was not included in our present study, no definitive conclusions could be drawn regarding the efficacy of MZR. However, the present treatment protocol did show a higher rate of $\mathrm{CR}$ at 12 months than the rates reported in previous studies, as well as continued efficacy for at least 24 months, even though the total dose of steroids we employed was considerably reduced and the duration of steroid therapy with additional use of MZR was also very short as compared with the current therapy without MZR.

None of the patients showed deterioration of renal function during the 24-month study period, and in fact patients with CKD stage 3 showed significant improvement of renal function. Since such good outcomes have not been reported for the current tonsillectomy-steroid pulse therapy, the improved efficacy was probably attributable to our additional use of MZR.

MZR is a selective inhibitor of inosine monophosphate (IMP) dehydrogenase, a rate-limiting enzyme in the de novo synthetic pathway of guanosine monophosphate (GMP). MZR selectively inhibits the proliferation of lymphocytes.

In addition to being a selective inhibitor of lymphocyte proliferation, MZR has been demonstrated to have some unique pharmacological properties. The 14-3-3 protein and heat shock protein 60 (HSP60) are known to be expressed in the glomeruli of patients with $\mathrm{IgAN}$. It has reported that MZR binds to the 14-3-3 protein [14] and HSP60 [15]. MZR enhances the transcriptional activity of the glucocorticoid receptor via binding to the 14-3-3 protein, suggesting that MZR might enhance the efficacy of steroids and contribute to steroid sparing [14].

The recovery of renal function in patients with renal hypofunction is also a relevant issue. It has been shown that MZR suppresses the infiltration of macrophages into the renal interstitium and the expression of $\alpha$-smooth muscle actin ( $\alpha$-SMA) in myofibroblasts [16]. In addition, MZR dose-dependently ameliorates renal tubulointerstitial fibrosis in rats with unilateral ureteral obstruction and significantly reduces the generation of osteopontin [10]. In IgAN patients who have received MZR treatment, a reduction in the number of $\mathrm{CD}^{+} 8^{+}$and $\alpha-\mathrm{SMA}^{+}$cells has also been reported [11]. Therefore, although we were unable to clarify the mechanism responsible for the effects of MZR on renal function, MZR appeared to contribute to renal recovery mainly by suppressing the infiltration of macrophages into the renal interstitium and the subsequent reduction of $\mathrm{CD}^{+} 8^{+}$and $\alpha-\mathrm{SMA}^{+}$cells.

IL-6 is produced by human mesangial and tubule cells, and its urinary levels have been shown to be correlated with the degree of mesangial proliferation in IgAN [17]. In the present study, a decrease in the urinary IL-6 level was observed in parallel with the response to therapy.

Since it is difficult to arrive at any definitive conclusions about whether the observed beneficial effects of the treatment were due to tonsillectomy or added MZR, a 
randomized controlled study will be required to compare tonsillectomy-steroid pulse therapy with and without MZR.

Although three patients developed adverse events during the follow-up period, all of these were mild. We consider that the safety of our therapeutic protocol is attributable to a lower incidence of adverse events related to MZR than to other immunosuppressive drugs. When MZR was used in combination with tonsillectomy, only one course of mPSL pulse therapy was sufficient, and the total dose of steroids administered was approximately half that used in the study by Hotta et al. Administration of MZR is considered to have reduced the incidence of adverse events that are typically associated with long-term steroid therapy. We plan to conduct further studies to assess the long-term outcomes of our therapeutic protocol.

In conclusion, tonsillectomy-steroid pulse therapy in combination with MZR appears to be safer than the current tonsillectomy-steroid pulse therapy alone for treatment of IgAN, and combination therapy with MZR holds promise for producing higher rates of CR. Our combination therapeutic protocol allows a reduction in the total dose of steroids, and is also recommended for patients with mild to moderate renal dysfunction.

Conflict of interest None declared.

\section{References}

1. Berger J, Hinglais N. Les dépôts intercapillaries ďIgA-IgG. J Urol Nephrol (Paris). 1968;74:694-5.

2. Chauveau D, Droz D. Follow-up evaluation of the first patients with IgA nephropathy described at Necker Hospital. Contrib Nephrol. 1993;104:1-5.

3. Pozzi C, Andrulli S, Del Vecchio L, Melis P, Fogazzi GB, Altieri $\mathrm{P}$, et al. Corticosteroid effectiveness in IgA nephropathy: longterm results of a randomized, controlled trial. J Am Soc Nephrol. 2004;15:157-63.

4. Hiki Y, Odani H, Takahashi M, Yasuda Y, Nishimoto A, Iwase $\mathrm{H}$, et al. Mass spectrometry proves under- $O$-glycosylation of glomerular IgA1 in IgA nephropathy. Kidney Int. 2001; 59:1077-85.
5. Hotta O, Miyazaki M, Furuta T, Tomioka S, Chiba S, Horigome I, et al. Tonsillectomy and steroid pulse therapy significantly impact on clinical remission in patients with $\operatorname{IgA}$ nephropathy. Am J Kidney Dis. 2001;38:736-43.

6. Komatsu H, Fujimoto S, Hara S, Sato Y, Yamada K, Kitamura K. Effect of tonsillectomy plus steroid pulse therapy on clinical remission of IgA nephropathy: a controlled study. Clin J Am Soc Nephrol. 2008;3:1301-7.

7. Yoshikawa N, Honda M, Iijima K, Awazu M, Hattori S, Nakanishi K, et al. Steroid treatment for severe childhood IgA nephropathy: a randomized, controlled trial. Clin J Am Soc Nephrol. 2006;1:511-7.

8. Shimizu M, Shou I, Tsuge T, Abe M, Tomino Y. Effect of mizoribine on glomerulonephritis of early-stage IgA nephropathy in ddY mice. Nephron. 1998;79:67-72.

9. Kawasaki Y, Suzuki J, Sakai N, Etoh S, Murai H, Nozawa R, et al. Efficacy of prednisolone and mizoribine therapy for diffuse IgA nephropathy. Am J Nephrol. 2004;24:147-53.

10. Sato N, Shiraiwa K, Kai K, Watanabe A, Ogawa S, Kobayashi Y, et al. Mizoribine ameliorates the tubulointerstitial fibrosis of obstructive nephropathy. Nephron. 2001;89:177-85.

11. Kawasaki Y, Hosoya M, Suzuki J, Onishi N, Takahashi A, Isome $\mathrm{M}$, et al. Efficacy of multidrug therapy combined with mizoribine in children with diffuse $\operatorname{Ig} \mathrm{A}$ nephropathy in comparison with multidrug therapy without mizoribine and with methylprednisolone pulse therapy. Am J Nephrol. 2004;24:576-81.

12. Tomino Y, Sakai H. Special Study Group (IgA Nephropathy) on Progressive Glomerular Disease. Clinical guidelines for immunoglobulin A ( $\operatorname{Ig} \mathrm{A})$ nephropathy in Japan, 2nd version. Clin Exp Nephrol. 2003;7:93-7.

13. Matsuo S, Imai E, Horio M, Yasuda Y, Tomita K, Nitta K, et al. Collaborators developing the Japanese equation for estimated GFR. Revised equations for estimated GFR from serum creatinine in Japan. Am J Kidney Dis. 2009;53:982-99.

14. Takahashi S, Wakui $\mathrm{H}$, Gustafsson JA, Zilliacus J, Itoh $\mathrm{H}$. Functional interaction of the immunosuppressant mizoribine with the 14-3-3 protein. Biochem Biophys Res Commun. 2000; 274:87-92.

15. Itoh $\mathrm{H}$, Komatsuda $\mathrm{A}$, Wakui $\mathrm{H}$, Miura $\mathrm{AB}$, Tashima $\mathrm{Y}$. Mammalian HSP60 is a major target for an immunosuppressant mizoribine. J Biol Chem. 1999;274:35147-51.

16. Sakai T, Kawamura T, Shirasawa T. Mizoribine improves renal tubulointerstitial fibrosis in unilateral ureteral obstruction (UUO)treated rat by inhibiting the infiltration of macrophages and the expression of $\alpha$-smooth muscle actin. J Urol. 1997;158:2316-22.

17. Dohi K, Iwano M, Muraguchi A, Horii Y, Hirayama T, Ogawa S, et al. The prognostic significance of urinary interleukin 6 in $\operatorname{IgA}$ nephropathy. Clin Nephrol. 1991;35:1-5. 